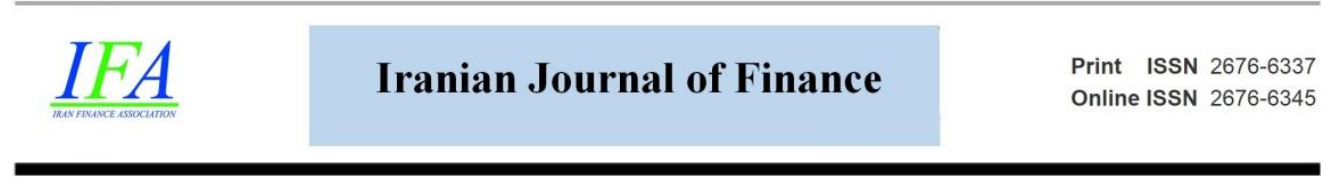

\title{
Stock Portfolio Optimization Using a Combined Approach of Relative Robust Risk Parity
}

\section{Sayed Mohammad Ebrahim Mirmohammadi}

Ph.D. Candidate, Faculty of Financial Engeenering, Kashan Branch, Islamic Azad University, Kashan, Iran. (Email: seb.mirmohammadi@gmail.com)

\section{Mehdi Madanchi zaj*}

*Corresponding Author, Assistant Prof., Department of Financial Management, Faculty of Management, E-Campus Branch, Islamic Azad University, Tehran, Iran. (Email: madanchi@iauec.ac.ir)

\section{Hossein Panahian}

Associate Prof., Department of Accounting, Kashan Branch, Islamic Azad University, Kashan, Iran. (Email: h.panahian@yahoo.com)

\section{Hossein Jabbary}

Assistance Prof., Department of Accounting, Kashan Branch, Islamic Azad University, Kashan, Iran. (Email: h.panasnjabbaryhian@yahoo.com)

Document Type: Original Article

Received: 2021/02/20

Accepted: 2021/08/11
2021, Vol. 5, No. 4. 87-106.

Published: 2021/15/05

\begin{abstract}
Risk parity is perceived as one of the stock portfolio selection models that have received a lot of attention since the US financial crisis in 2008. The philosophy of this model is to allocate the same amount of portfolio risk between the constituent assets. In the present study, the combined portfolio selection model of relative robust risk parity is introduced, which uses the worst-case scenario approach on the covariance matrix parameter appearing in the robust risk model in portfolio robustness. According to historical data, several scenarios are considered for the covariance matrix. The objective function value of the hybrid model for each portfolio (feasible point) is the worst result (with most volatility) among the set of scenarios. Finally, the model selects a portfolio for
\end{abstract}


which the worst possible result has the least relative volatility. The research portfolio consists of 8 industries from Tehran Stock Exchange in the period 2011 to 2020. This portfolio has a higher Sharpe ratio than conventional models of mean-variance and weight parity, and is more resilient to market declines than the two models and produces less loss. Therefore, risk-averse investors are advised to use this stock portfolio selection model as a cover to face severe market declines.

Keywords: Risk parity portfolio, Relative robustness, Sharpe ratio, Particle Swarm Optimization algorithm

DOI: https://doi.org/ 10.30699/IJF.2021.269599.1193 Publisher: Iran Finance Association

Copyright: author(s) Type of License: Creative Commons License (CC-BY 4.0)

\section{Introduction}

The stock portfolio has an expected return and risk at the time of design. These values are a function of the return and risk of each asset in the portfolio. The expected return on the portfolio is often the weighted average of the individual returns on assets where the weight of each asset (numerically between zero and one) is equal to the percentage of its share of the initial capital. Portfolio risk, on the other hand, is more complexly dependent on the risk of its constituent elements. For example, if the variance measure is used for risk, in addition to the variance of individual assets, all correlations between asset pairs must be considered. By creating an optimal portfolio, the investor intends to reach the expected return with the least possible risk. Therefore, an efficient portfolio with a certain level of return has the least risk. Many models have been developed for the optimal stock portfolio selection problem, and the Markowitz portfolio model (mean-variance model) was the first in this regard. Various models of stock portfolio selection differ greatly in terms of the objective function, limitations, single-period or multi-period, how the parameters are approximated, and so on.

Due to the uncertainty of the parameters in the stock portfolio selection models ( especially in Iran, the capital market is always faced with many risks, especially political risk and changes in the rules, which can expose investors' portfolio assets to unforeseen sharp price changes and possibly lead to sharp declines), if the values that are seen realistically and objectively after closing the portfolio in the market deviate significantly from the estimated values, the portfolio return takes considerable distance from what is expected. This can lead to large and unpredictable losses. In fact, the portfolio faces a lack of 
balance and robustness, and to reduce this consequence, the present study presents a combined portfolio model of relative robust risk parity.

In this hybrid model, the purpose of the risk parity portfolio is to equalize the share of asset risk in the portfolio risk, but this risk division is a statistical operation due to correlation matrix parameter and the purpose of relative robust is to ensure equal sharing of risk in different scenarios. The study of the research background shows that the robustness of the risk parity portfolio on the parameter of covariance matrix has not been modeled so far and the present study provides relative robustness for the risk parity portfolio. The structure of the article includes the theoretical foundations, research background, research method, research findings and conclusions.

\section{Theoretical Foundations}

Each asset has a share of portfolio risk. If one or more assets whose share of risk is higher than the total portfolio risk deviate from their expected return, the return on the portfolio will take considerable distance from the expected return. Especially if in reality one or a number of these stocks face a severe decline, the portfolio may confront a large loss, too. The risk parity portfolio is based on a fundamental principle, which is to select the optimal portfolio based on the allocation of as much equal risk as possible between assets. In other words, in this approach, the share of asset risk out of the total portfolio risk should be as equal as possible. This same allocation is in fact a kind of hedge for the portfolio in order to deal with scenarios of a sharp decline in the future return on assets in the portfolio.

Risk parity is an approach to managing an investment portfolio that focuses on risk allocation (meaning instability) rather than capital allocation. According to this approach, when asset allocation is done at the level of risk parity, the constructed portfolio is expected to have more Sharpe ratio and be more resilient to market decline than the traditional portfolio (Roncalli \& Weisang, 2016). For example, the research by Demigueil et al. (2008) as well as Chaves et al. (2012) shows that with regard to the Sharpe ratio, risk parity often has better results compared to the optimal mean-variance portfolio strategy. The asset allocation approach with equal risk parity was first presented by Edward Qjan in 2005 in an article on asset management. After a while, this strategy came to the attention of Asset Management. Some theoretical aspects of this approach were developed between 1950 and 1970, but the first fund based on the risk parity approach, called the All-Weather, was established in 1996. In recent years, many mutual funds have recommended risk parity funds to their clients. This approach was also used in 2005 and then 
adapted to the asset management industry. Since then, many investment companies, asset management and funds such as Aquila Capital, North Water, Wellington, etc. have used this approach.

According to a 2015 article in the Wall Street Journal with the title " Are Risk-Parity Funds a Better Strategy for Diversification ", funds with a balanced risk approach performed relatively well during the 2008 financial crisis. For example, during the same period, the IQR fund with the balanced risk approach fell only $18 \%$, which was better than the $22 \%$ decline of the Vanguard balanced index fund. Also, the types of balanced risk funds offered by hedge funds have been steadily increasing in popularity and have produced expected returns since the financial crisis.

In most optimization problems (in general), there are coefficients and parameters that need to be estimated. In some cases, these coefficients are quite clear. For example, if a coefficient is the price of a product, it can be estimated with high certainty based on market price data. In the meantime, there are models whose coefficients and parameters cannot be obtained with certainty and statistical estimation is often used for them. For example, in the portfolio selection model, expected returns and asset risk cannot be estimated with certainty because the market will build them in the future. This is the case where robust optimization enters. Its purpose is optimization with the aim of increasing confidence in the expected results in the theoretical and constructing stage of the portfolio. Robust optimization is a practical solution to problems in which the amount and distribution of parameters are not certain. Various methods have been defined to solve problems using robust optimization. One of the most common of these is the worst-case scenario approach.

In this approach, several possible scenarios for the uncertain parameter are considered. Then for each feasible value of the response space, the objective function is calculated for all scenarios and the result of optimization in that feasible value is reported as the worst result of the scenarios. Finally, some feasible points which have the best value of the objective function, are returned as the optimization answer. In summary, the answer is chosen from which the worst possible result (among scenarios) has the least loss (the most beneficial). The most important drawback of this method is that it is cautious (in fact, this approach does not effectively exploit the possibility of the occurrence of other scenarios), but will be very useful in decision making. Therefore, the assurance is established as follows: We do not know which scenario will happen, so let's calculate the worst answer in each scenario and choose the best one. 
The approach of the present study is to select the optimal portfolio of relative robust risk parity. The purpose of this work is to reassure the investor about the practical and real results of the theoretical portfolio. Consider an optimization model with several scenarios for one uncertain parameter. Optimizing the model with a specific scenario leads to a minimum loss, which can be considered as the best capability of that scenario. Now in relative robust optimization, the performance of a feasible point is identified good if it can approach the least losses that scenarios can produce. Relative robust optimization helps to ensure that the worst-case scenario is taken into account and risk parity helps to avoid drastic changes in scenarios that are not considered and that the market will create. Said that the main research questions are as follows:

1. Is the stock portfolio selection model of relative robust risk parity in the Sharpe ratio criterion better than conventional Markowitz and weight parity models?

2. Is the stock portfolio selection model of relative risk-based parity more robust than the conventional Markowitz and weight parity models in severe market losses? .

\section{Research Background}

In what follows, a sketch of the literature review will be presented. Davallou et al. (2017) in a study compared the performance of three stock portfolio strategies of mean-variance, weight parity and risk parity in terms of risk, return and Sharpe ratio. The sample includes weekly data of 25 main Tehran Stock Exchange indexes from 2006 to the beginning of 2016. The results of this study show that the asset allocation strategy based on equal risk share in most cases has the middle performance and in some cases has the best performance compared to the other two strategies. Thus, by using this approach, investors and portfolio managers will have a more reliable performance. Rezaei et al. (2017) examined the optimal robust portfolio model using the objective function of expected shortfall. The results show that if the level of conservatism increases, the value of the objective function will increase. NamdarZanganeh and Hassanpour (2017) presented a new robust model based on the Min/Max goal programming approach for the problem of multi-objective portfolio selection. The results show that the modeling can be well suited to deal with uncertainty in the problem of determining the multiobjective financial portfolio. Gharakhani et al. (2016) proposed a robust optimization approach to solve the problem of multi-period portfolio selection. 
In this study, in order to estimate the expected return on assets, the capital asset pricing model has been used. Jafari and Khajehzadeh (2015) presented a fuzzy robust multi-objective model. The results show the efficiency of the proposed method in terms of considering risk, return, budget and investment limits per share.

Costa and Kwon (2018) modeled stock portfolio selection with an equal risk approach between portfolio elements. To model returns, they used the Markov regime-switching model to be able to control the sharp changes in returns caused by economic cycles. The results of the optimization of a diversified portfolio with 50 stocks between 2000 and 2010 show that portfolio performance in out-of-sample data is better on the Sharpe ratio than the Markowitz model and the model without Markov switching modeling. Roncalli and Weisang (2016) decomposed the risk of a portfolio according to its constituent elements and extended the issue of stock portfolio selection to the diversification of decomposition risk between portfolio elements. They applied the method used in several sample cases, including asset allocation using the Fama and French factor model, asset selection in hedge funds, and strategic asset allocation based on economic factors. Chaves et al. (2010) conducted their research on long-term bonds, securities with different investment ratings, emerging stock markets, commodities, and land and building funds. They found that a portfolio strategy with an equal share of risk had a better Sharpe ratio than the minimum variance method. But this is not the case with portfolios with equal weighting and 60-40 retirement portfolios.

Maillard et al. (2009) conducted research on 10 US industry indices from 1973 to 2008. They found that in the mentioned period, the performance of the portfolio strategy with an equal share of risk in terms of return, risk, Sharpe ratio, diversification and maximum capital loss is between the other two methods. Lee (2010) in his research on S\&P 1500 stocks showed that riskbased allocation does not necessarily provide a better return on risk than other methods. In a study by Caporin et al. (2012) on 30 large stocks in each of the US, European and Japanese markets, they showed that the optimal performance of a portfolio strategy with equal risk share in terms of risk, return, Sharpe ratio, maximum capital loss and diversification has led more investment managers to use this method. Simoes et al. (2018) modeled the problem of optimal stock portfolio selection based on the robust optimization approach. In this model, the uncertain parameter is the covariance matrix for which different scenarios are considered. The regret benchmark, which measures maximum volatility and risk, has also been defined and added to the model. Wozabal and Pflug (2016) created a problem in a robust portfolio selection in which the 
confidence set described the possibility of distributing the return on assets. They also assessed the relationships between risk, robustness, and portfolio return. Their results showed that when stability increases, the risk and return of the portfolio decreases and the portfolio becomes more diversified. Boyd and Kim (2015) with a separable set of uncertainties developed several different methods for modeling a set of uncertainties for the expected returns vector and covariance matrices. Gregory et al. (2016) presented a model that minimizes the difference between the estimated and actual impact of factors on maximizing portfolio returns. The results showed that minimizing this distance brings the estimates closer to the correct boundaries and the robust model often results in more realistic returns. Bai et al. (2015) in optimizing risk parity portfolio used the non-convex least-squares optimization approach to optimize the portfolio of least variance in which the obtained answers are equal to the sum of all optimal answers of risk parity portfolio. They showed that the answer obtained from the algorithm is very close to the optimal answer.

Marat (2020) introduced a hierarchical risk parity portfolio model that uses an exponentially weighted covariance matrix and places constraints on the model for further variability. The results of the research using Monte Carlo simulation show that the estimated model reduces downside risk by up to fifty percent. Gambetta and Vaughn (2020) introduced a relaxed risk parity portfolio selection model. In this model, the investor can add a certain minimum level of return as a constraint to the problem. The practical result of the research shows that the introduced portfolio has maintained the conservatism ability of the risk parity portfolio. Fabio et al. (2019) considered expectiles as risk measures for practically finding risk parity portfolios and compared the accuracy and efficiency of these methods on real-world data. Mohammadi and Mohammadi (2018) incorporate future returns scenarios in the investment decision process. In order to representative points on the efficient frontier, the minimax regret (relative robust) portfolio is calculated, on the basis of the aforementioned scenarios. In this way, the areas of the efficient frontier that are more robust than others are identified. The main contribution in this paper is related to the extension of the conventional minimax regret criterion formulation, in multiobjective programming problems. The validity of the proposed approach is verified through an empirical testing application on the top 75 companies of the Tehran Stock Exchange Market in 2017. Groetzner and Werner (2021) extend the concept of regret from the single-objective case to the multi-objective setting and introduce a proper definition of multivariate relative regret. In contrast to the few existing ideas that mix scalarization and optimization, they clearly separate the modelling of multi-objective (robust) regret from its numerical solution. Moreover, this approach is not limited to a finite 
uncertainty set or interval uncertainty and computations or at least approximations remain tractable in several important special cases. They illustrate all approaches based on a bi-objective shortest path problem under uncertainty.

According to the history of studies, the innovation of the present study is the use of relative robust optimization on the covariance matrix parameter in the risk parity portfolio. This relative robustness, which is done with the view of the worst-case scenario, helps the risk parity portfolio in two cases. The first is that if the covariance matrix deviates from the expected values of the stock portfolio, it should not be subject to drastic changes in portfolio outputs, and the second is that the relativity helps each scenario to be used to its own capabilities.

\section{Research Methodology}

Suppose we have a portfolio with $\mathrm{n}$ assets and weight vector $w$. The standard deviation of the portfolio return, as a measure of risk, is then equal to $\sigma_{P}=$ $\sqrt{w^{t} \sum w}$, which $\Sigma$ stands for the asset covariance matrix. If $\sigma_{i} 1 \leq i \leq n$ is the share of the risk of each asset of portfolio risk ( $\sigma_{i}$ does not indicate a standard deviation here) then the following equation can be written:

$\sigma_{P}=\sqrt{w^{t} \sum w}=\sum_{i=1}^{n} \sigma_{i}$

On the other hand, according to the differential relationship $d \sigma_{P}==\sum_{i=1}^{n} w_{i} \frac{\partial \sigma_{P}}{\partial w_{i}}$, the share of the risk of asset $i$ is equal to $\sigma_{i}=w_{i} \frac{\left(\sum w\right)_{i}}{\sqrt{w^{t} \Sigma w}}$. The risk parity model seeks to make equal the share of assets risk out of the total risk of the portfolio and for this purpose optimizes the equation (2). In equation (2), due to the positive factors that exist in the objective function, in order to minimize, all factors should be as close as possible, and this means that all the share of risks or $\sigma_{i}$ should be as close as possible.

$\min \sum_{i=1}^{n} \sum_{j=1}^{n}\left(w_{i}\left(\sum w\right)_{i}-w_{j}\left(\sum w\right)_{j}\right)^{2}$

$1^{t} w=1$

$w \geq 0$

$w \geq 0$ This shows that short selling is not part of the model assumptions. The 
form equivalent to the problem of risk parity is the equation (3):

$$
\begin{aligned}
& \min _{\psi} \psi^{t} \sum \psi \\
& \sum_{\psi} \operatorname{Ln}\left(\psi_{i}\right) \geq C
\end{aligned}
$$

Where $C$ is an arbitrary constant (Costa and Kwon,2018)? desired positive value and optimal risk parity portfolio weights are determined by normalizing the values $\psi_{i}$ as $w_{i}^{*}=\frac{\psi_{i}}{\sum_{i=1}^{n} \psi_{i}}($ Costa and Kwon, 2018). Equation 3 is an essential representation of the risk parity portfolio that allows us to define relative robustness on the covariance matrix parameter. As can be seen, an important parameter in risk parity is the covariance matrix. Expecting a statistical estimate of a parameter in the stock portfolio and its long-term use is at odds with its dynamic nature. To solve this problem, a set of possible scenarios for the covariance matrix, the $U$ set, can be taken into account. This set includes several matrices, each of which is a probable scenario for the future of the covariance matrix of returns on assets in the portfolio. Considering the set of possible scenarios, the stock portfolio selection problem can be turned into an equation

$$
\begin{gathered}
\min _{\psi} \max _{\Sigma \in U} \psi^{t} \sum \psi \\
\sum_{\psi \geq 0} \operatorname{Ln}\left(\psi_{i}\right) \geq C
\end{gathered}
$$

The approach used in this model is robust optimization with the worstcase scenario. The value of the objective function of this model for each portfolio (feasible point) is the worst result (with most volatility) among the set of scenarios, and finally, the model selects the portfolio for which the worst possible result has the least amount of risk. The view used in this model is an absolute one in which the worst-case scenario is used and thus the other existing scenarios and the probabilities of their occurrence are not used much. In the next step, the concept of the worst-case scenario is defined relatively according to equation (5).

Each vector of weights for the portfolio can be combined with different scenarios of the covariance matrix to produce a value of the objective function (here risk). Then the optimal portfolio has the least loss among all vectors. This means that we compare the maximum losses produced for vectors in different scenarios in an absolute way. However, Optimizing the portfolio with each 
scenario of the covariance matrix results in a minimum loss and therefore has a maximum capability (minimum risk). Relative robust optimization tries to compare the worst-case scenario results according to their capabilities. An example is given to clarify the relative concept. Consider two students. The first student scored 14 marks and the second one scored 15 marks. The first student has not scored better than 15 so far (the highest score he/she can get), while the second student has not scored better than 18. From the absolute point of view, the second student outperforms the first one $(14<15)$ and from the relative point of view, the first student has a better result $(15-18<14-15)$. The main idea for a relatively robust model is taken from Simoes et al. (2018). According to this example, the relative form of equation (4) is:

$$
\begin{aligned}
& \min _{\psi} \max _{\Sigma \in U}\left(\sqrt{\psi^{t} \sum \psi}-\min _{y} \sqrt{y^{t} \sum y}\right) \\
& \sum_{\boldsymbol{L}} \operatorname{Ln}\left(\psi_{i}\right) \geq C \\
& \sum_{\psi} \operatorname{Ln}\left(y_{i}\right) \geq C \\
& 0, y \geq 0
\end{aligned}
$$

The model in this paper is called the relative robust risk parity. In fact, each scenario from its own set of scenarios can be used as a covariance matrix in the risk parity model and produce an optimal solution. This optimal solution shows that any other portfolio formed with this covariance matrix in the parity model will have a larger (worse) objective function. Therefore, for each $\sum \in U$ and $\psi$, $\left(\sqrt{\psi^{t} \sum \psi}-\min _{y} \sqrt{y^{t} \sum y}\right)$ is positive and is a relative distance in which each scenario or matrix is measured by its own capabilities. To optimize equation (5), the Particle Swarm Optimization algorithm in MATLAB software will be used. The Particle Swarm Optimization Algorithm (PSO) is a population-based social search algorithm modeled on the social behavior of birds and fishes.

\section{Research Findings}

The research portfolio consists of 8 indices or industries from Tehran Stock Exchange in the period 2011 to 2020. Using indices means forming a diversified portfolio of stocks in that industry. For example, using the car index as an asset means buying a subset of this index in a variety of ways (commensurate with their weight in the index). The time horizon of the portfolio is one week (lasts for a period of one week) and 5 working days are considered every week. Descriptive statistics for 432 weekly returns on assets are presented in Table (1). Each asset has a number that will be referred to 
Stock Portfolio Optimization Using a Combined Approach of...

from now on.

Table 1. Descriptive statistics of weekly return on portfolio assets

\begin{tabular}{|c|c|c|c|c|c|c|}
\hline $\begin{array}{c}\text { Statistical } \\
\text { index } \\
\text { asset }\end{array}$ & $\begin{array}{c}\text { Asset } \\
\text { No. }\end{array}$ & mean & median & min & max & $\begin{array}{c}\text { Standard } \\
\text { deviation }\end{array}$ \\
\hline bank & 1 & 0.005501 & 0.00006 & -0.1071 & 0.210828 & 0.037413796 \\
\hline automobile & 2 & 0.006629 & -0.00029 & $\begin{array}{c}- \\
0.15248\end{array}$ & 0.235092 & 0.056627454 \\
\hline Metal ores & 3 & 0.008335 & -0.00109 & -0.1192 & 0.204428 & 0.044456103 \\
\hline $\begin{array}{c}\text { blocks of } \\
\text { cement }\end{array}$ & 4 & 0.008374 & -0.00042 & -0.1308 & 0.180779 & 0.039458241 \\
\hline oil & 5 & 0.009714 & 0.002594 & $\begin{array}{c}- \\
0.41696\end{array}$ & 0.36132 & 0.052053383 \\
\hline machinery & 6 & 0.010098 & 0.003865 & $\begin{array}{c}- \\
0.12877\end{array}$ & 0.220871 & 0.0402561 \\
\hline drugs & 7 & 0.014966 & -0.0013 & $\begin{array}{c}- \\
0.74295\end{array}$ & 2.855466 & 0.176013222 \\
\hline sugar & 8 & 0.011543 & 0.002834 & $\begin{array}{c}- \\
0.15822\end{array}$ & 0.249305 & 0.050472704 \\
\hline
\end{tabular}

432 weekly return data are divided into 360 data for training and approximation of stock portfolio selection model parameters and 72 data for portfolio testing and evaluation. The training data were divided into 3 groups of 120 members and each group was used to calculate one of the covariance matrix scenarios. Therefore, three scenarios were designed for the asset covariance matrix, which is presented in tables (2) to (4) in the form of a correlation coefficient matrix.

Table 2. The first scenario for the correlation coefficient matrix

\begin{tabular}{|l|l|l|l|l|l|l|l|l|}
\hline Asset No. & $\mathbf{1}$ & $\mathbf{2}$ & $\mathbf{3}$ & $\mathbf{4}$ & $\mathbf{5}$ & $\mathbf{6}$ & $\mathbf{7}$ & $\mathbf{8}$ \\
\hline 1 & 1.000 & 0.555 & 0.323 & 0.319 & 0.127 & 0.424 & -0.031 & 0.050 \\
\hline 2 & 0.555 & 1.000 & 0.285 & 0.282 & 0.139 & 0.477 & -0.040 & 0.199 \\
\hline 3 & 0.323 & 0.285 & 1.000 & 0.158 & 0.306 & 0.322 & -0.069 & -0.014 \\
\hline 4 & 0.319 & 0.282 & 0.158 & 1.000 & 0.071 & 0.379 & -0.061 & 0.044 \\
\hline 5 & 0.127 & 0.139 & 0.306 & 0.071 & 1.000 & 0.187 & -0.035 & -0.068 \\
\hline 6 & 0.424 & 0.477 & 0.322 & 0.379 & 0.187 & 1.000 & 0.021 & 0.058 \\
\hline 7 & -0.031 & -0.040 & -0.069 & -0.061 & -0.035 & 0.021 & 1.000 & 0.149 \\
\hline
\end{tabular}


Iranian Journal of Finance, 2021, Vol. 5, No. 4 (Mirmohammadi, S.)

\begin{tabular}{|l|l|l|l|l|l|l|l|l|}
\hline 8 & 0.050 & 0.199 & -0.014 & 0.044 & -0.068 & 0.058 & 0.149 & 1.000 \\
\hline
\end{tabular}

Table 3. The second scenario for the correlation coefficient matrix

\begin{tabular}{|c|c|c|c|c|c|c|c|c|}
\hline Asset No. & $\mathbf{1}$ & $\mathbf{2}$ & $\mathbf{3}$ & $\mathbf{4}$ & $\mathbf{5}$ & $\mathbf{6}$ & $\mathbf{7}$ & $\mathbf{8}$ \\
\hline 1 & 1.000 & 0.647 & 0.398 & 0.422 & 0.088 & 0.363 & 0.141 & 0.177 \\
\hline 2 & 0.647 & 1.000 & 0.413 & 0.485 & 0.237 & 0.504 & 0.094 & 0.219 \\
\hline 3 & 0.398 & 0.413 & 1.000 & 0.382 & 0.287 & 0.386 & 0.083 & 0.110 \\
\hline 4 & 0.422 & 0.485 & 0.382 & 1.000 & 0.215 & 0.626 & 0.104 & 0.249 \\
\hline 5 & 0.088 & 0.237 & 0.287 & 0.215 & 1.000 & 0.199 & -0.006 & -0.057 \\
\hline 6 & 0.363 & 0.504 & 0.386 & 0.626 & 0.199 & 1.000 & 0.040 & 0.281 \\
\hline 7 & 0.141 & 0.094 & 0.083 & 0.104 & -0.006 & 0.040 & 1.000 & -0.072 \\
\hline 8 & 0.177 & 0.219 & 0.110 & 0.249 & -0.057 & 0.281 & -0.072 & 1.000 \\
\hline
\end{tabular}

Table 4. The third scenario for the correlation coefficient matrix

\begin{tabular}{|c|c|c|c|c|c|c|c|c|}
\hline Asset No. & $\mathbf{1}$ & $\mathbf{2}$ & $\mathbf{3}$ & $\mathbf{4}$ & $\mathbf{5}$ & $\mathbf{6}$ & $\mathbf{7}$ & $\mathbf{8}$ \\
\hline 1 & 1.000 & 0.351 & 0.179 & 0.394 & 0.204 & 0.315 & 0.305 & 0.168 \\
\hline 2 & 0.351 & 1.000 & 0.194 & 0.459 & 0.140 & 0.473 & 0.273 & 0.311 \\
\hline 3 & 0.179 & 0.194 & 1.000 & 0.216 & 0.555 & 0.143 & 0.018 & 0.026 \\
\hline 4 & 0.394 & 0.459 & 0.216 & 1.000 & 0.310 & 0.642 & 0.304 & 0.298 \\
\hline 5 & 0.204 & 0.140 & 0.555 & 0.310 & 1.000 & 0.232 & 0.091 & 0.149 \\
\hline 6 & 0.315 & 0.473 & 0.143 & 0.642 & 0.232 & 1.000 & 0.266 & 0.326 \\
\hline 7 & 0.305 & 0.273 & 0.018 & 0.304 & 0.091 & 0.266 & 1.000 & 0.137 \\
\hline 8 & 0.168 & 0.311 & 0.026 & 0.298 & 0.149 & 0.326 & 0.137 & 1.000 \\
\hline
\end{tabular}

With the set of scenarios available, equation (5) i.e., the model of relative robust risk parity was optimized using particle Swarm Optimization algorithm with 200 Particles and 1000 replications in MATLAB software and the optimal portfolio is presented in Table (5).

Table 5. Optimal portfolio of relative robust risk parity model

\begin{tabular}{|c|c|c|c|c|c|c|c|c|}
\hline asset & $\begin{array}{c}\text { bank } \\
\mathrm{s}\end{array}$ & $\begin{array}{c}\text { automobil } \\
\mathrm{es}\end{array}$ & $\begin{array}{c}\text { Metal } \\
\text { ores }\end{array}$ & $\begin{array}{c}\text { cemen } \\
\mathrm{t}\end{array}$ & oil & $\begin{array}{c}\text { types of } \\
\text { machinery }\end{array}$ & drugs & sugar \\
\hline $\begin{array}{c}\text { weigh } \\
\mathrm{t}\end{array}$ & $\begin{array}{c}0.14 \\
4\end{array}$ & 0.075 & 0.142 & 0.176 & $\begin{array}{c}0.12 \\
5\end{array}$ & $0 . .135$ & $\begin{array}{c}0.037 \\
5\end{array}$ & $\begin{array}{c}0.161 \\
2\end{array}$ \\
\hline
\end{tabular}


After being in closed form, the stock portfolios were evaluated on 72 test

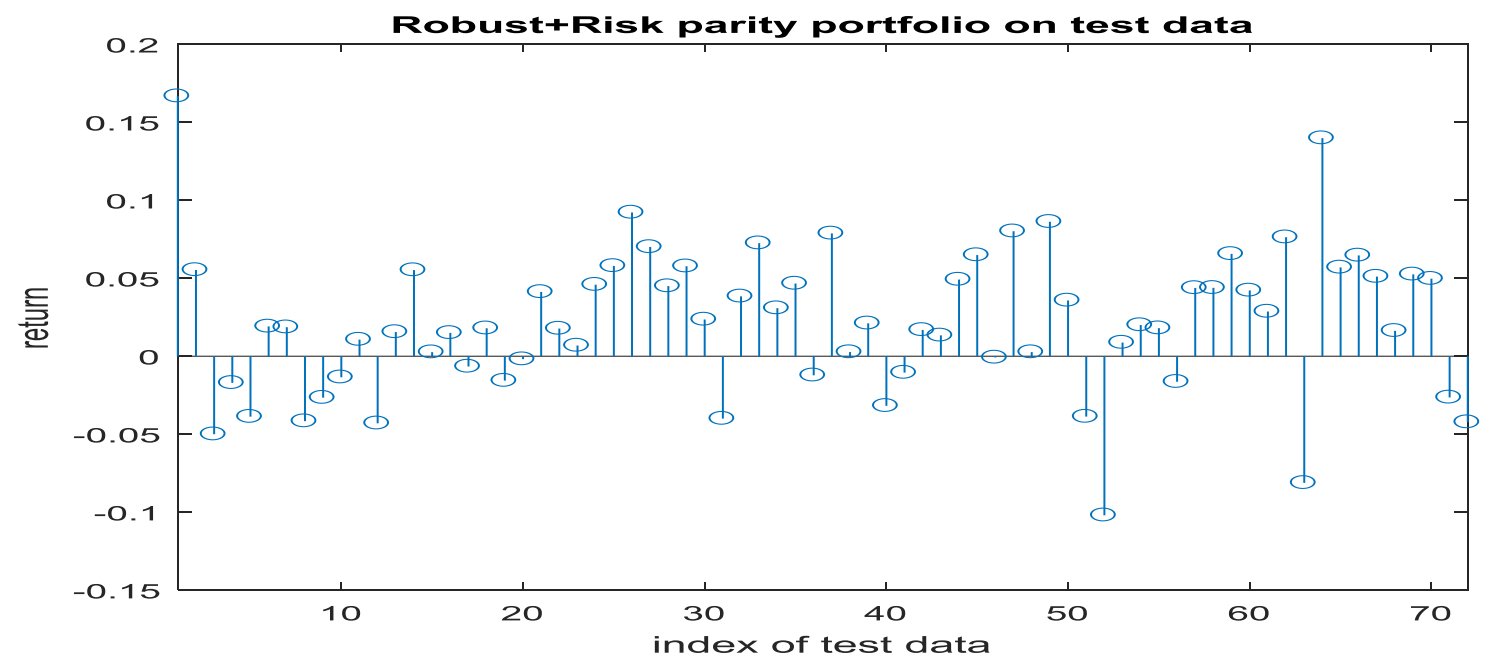

data. The weekly returns obtained are presented in Figure (1).

Figure 1. Returns from the model of relative robust risk parity on test data

According to the returns obtained on 72 test data, the portfolio performance is presented based on the average weekly return, risk and weekly Sharpe ratio in Table (6). The Sharp ratio is calculated by dividing the return by the risk.

Table 6. Relative robust risk parity portfolio performance on test data

\begin{tabular}{|c|c|}
\hline index & value \\
\hline Average weekly return & 0.0231 \\
\hline Weekly risk( standard deviation) & 0.0458 \\
\hline Sharpe ratio & 0.5047 \\
\hline
\end{tabular}

According to Table (6), from this portfolio, one can expect an average weekly return of 0.0231 while taking a risk of 0.0458 . Sharpe ratio also shows that for each higher risk unit, a reward of 0.5047 can be received.

In the following, we evaluate the performance of a portfolio with equal weighting and the portfolio of weight parity. In this portfolio, without any 
optimization, the share of each asset is equal and is $1 / 8$. This portfolio was evaluated on 72 test data, the returns of which are presented in Figure (2).

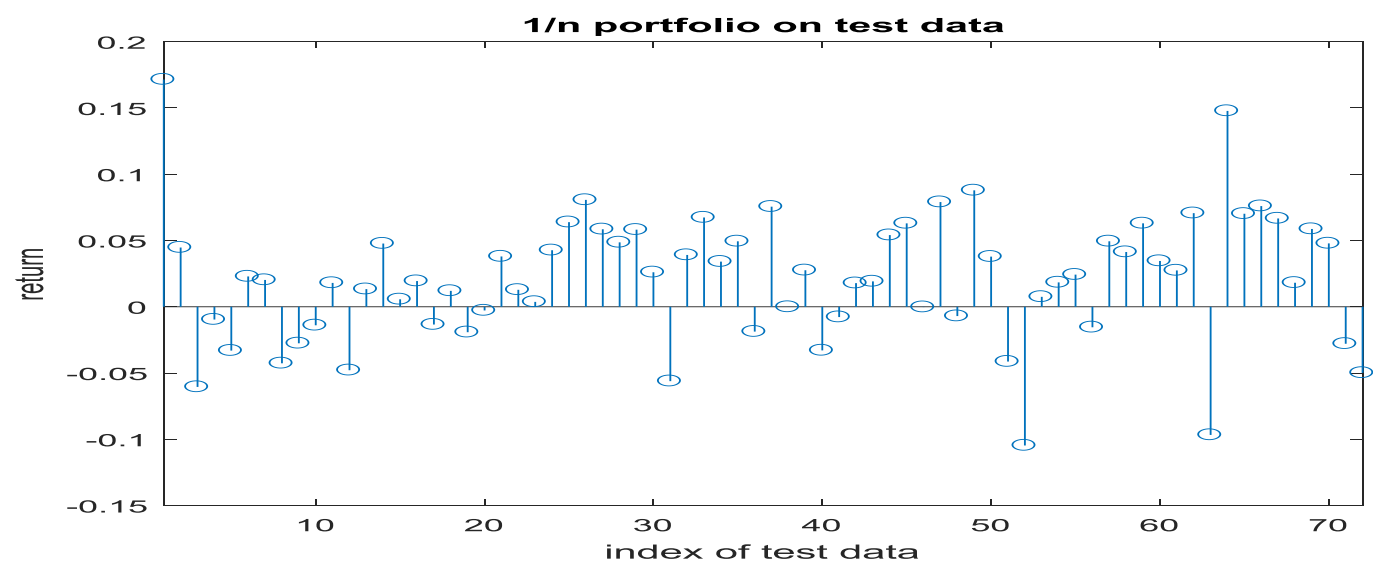

Figure 2. Return of weight parity portfolio on test data

According to the returns obtained on 72 test data, the portfolio performance is presented in Table (7).

Table 7. Weight parity portfolio performance on test data

\begin{tabular}{|c|c|}
\hline index & value \\
\hline Average weekly return & 0.0216 \\
\hline Weekly risk(standard deviation) & 0.0482 \\
\hline Sharpe ratio & 0.4481 \\
\hline
\end{tabular}

According to Table (7), from this portfolio, one can expect an average weekly return of 0.0216 while taking a risk of 0.0482 . Sharpe ratio also shows that for each higher risk unit, a reward of 0.4481 can be received.

The next portfolio is the mean-variance or Markowitz model. The objective function in this model is to minimize variance. All 360 training data were used to approximate the covariance matrix. The optimal weights of this portfolio were calculated with the help of quadratic optimization in MATLAB software and are presented in table (8).

Table 8. the optimal portfolio of the Markowitz model

\begin{tabular}{|c|c|c|c|c|c|c|c|c|}
\hline asset & $\begin{array}{c}\text { bank } \\
\text { s }\end{array}$ & $\begin{array}{c}\text { automobil } \\
\text { es }\end{array}$ & $\begin{array}{c}\text { Metal } \\
\text { ores }\end{array}$ & $\begin{array}{c}\text { cemen } \\
\text { t }\end{array}$ & oil & $\begin{array}{c}\text { types of } \\
\text { machinery }\end{array}$ & $\begin{array}{c}\text { drug } \\
\text { s }\end{array}$ & $\begin{array}{c}\text { suga } \\
\mathbf{r}\end{array}$ \\
\hline
\end{tabular}




\begin{tabular}{|c|c|c|c|c|c|c|c|c|}
\hline $\begin{array}{c}\text { weig } \\
\text { ht }\end{array}$ & 0.287 & 0 & 0.087 & 0.294 & $\begin{array}{c}0.08 \\
2\end{array}$ & 0.101 & $\begin{array}{c}0.00 \\
7\end{array}$ & $\begin{array}{c}0.13 \\
8\end{array}$ \\
\hline
\end{tabular}

After being in closed form, this portfolio was evaluated on 72 test data,

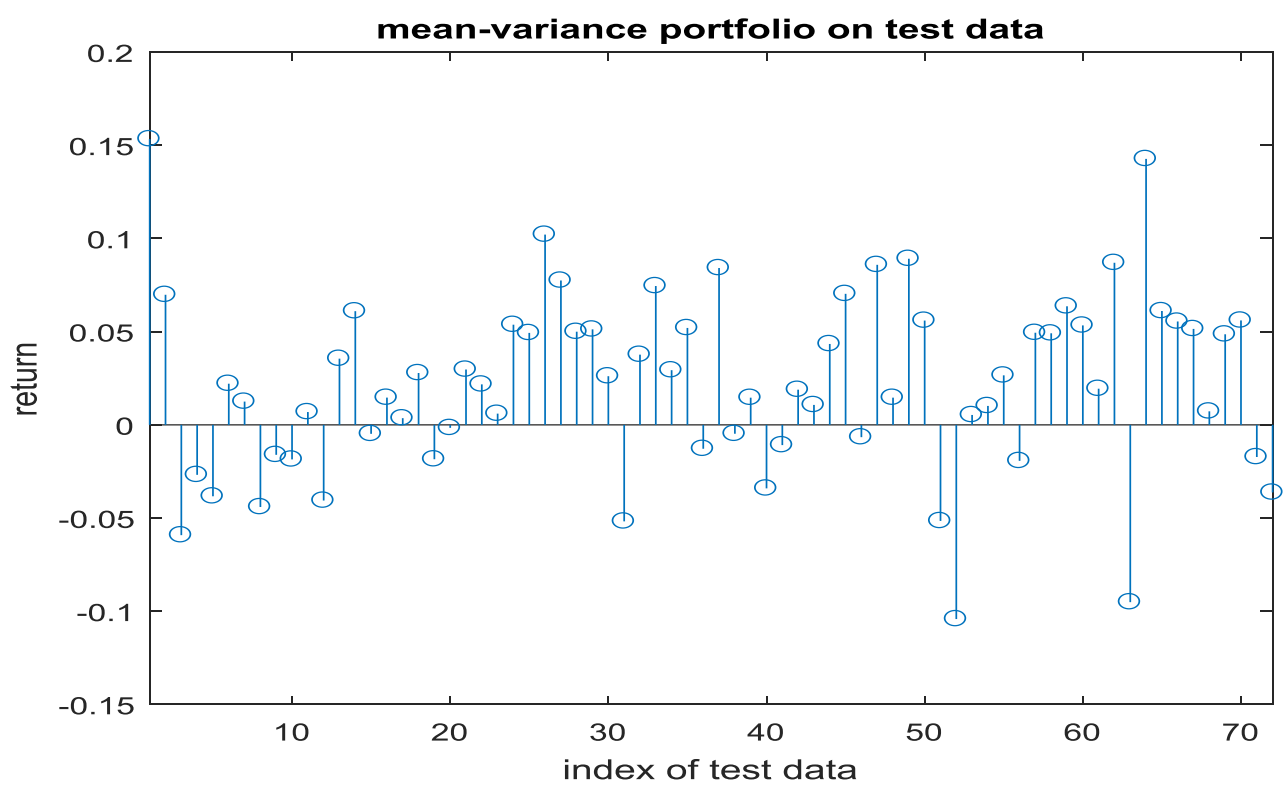

the returns of which are presented in Figure (3).

Figure 3. Markowitz portfolio returns on test data

According to the returns obtained on 72 test data, the portfolio performance is presented in Table (9).

Table 9. Markowitz portfolio performance on test data

\begin{tabular}{|c|c|}
\hline index & value \\
\hline Average weekly return & 0.0226 \\
\hline Weekly risk(standard deviation) & 0.048 \\
\hline Sharpe ratio & 0.4708 \\
\hline
\end{tabular}

According to Table (9), from this portfolio, one can expect an average weekly return of 0.0226 while taking a risk of 0.048 . Sharpe ratio also shows that for each higher risk unit, a reward of 0.4708 can be received.

As can be seen from the comparison of the performance tables of the 
three models, the Sharpe ratio of the introduced hybrid model is higher than the other two models and in this respect, it has a better performance. As stated in the theoretical foundation section, one of the advantages of risk parity models is their greater resistance to market shortfalls and is expected to produce less loss. The chart below shows the performance of three portfolios on 72 test data.

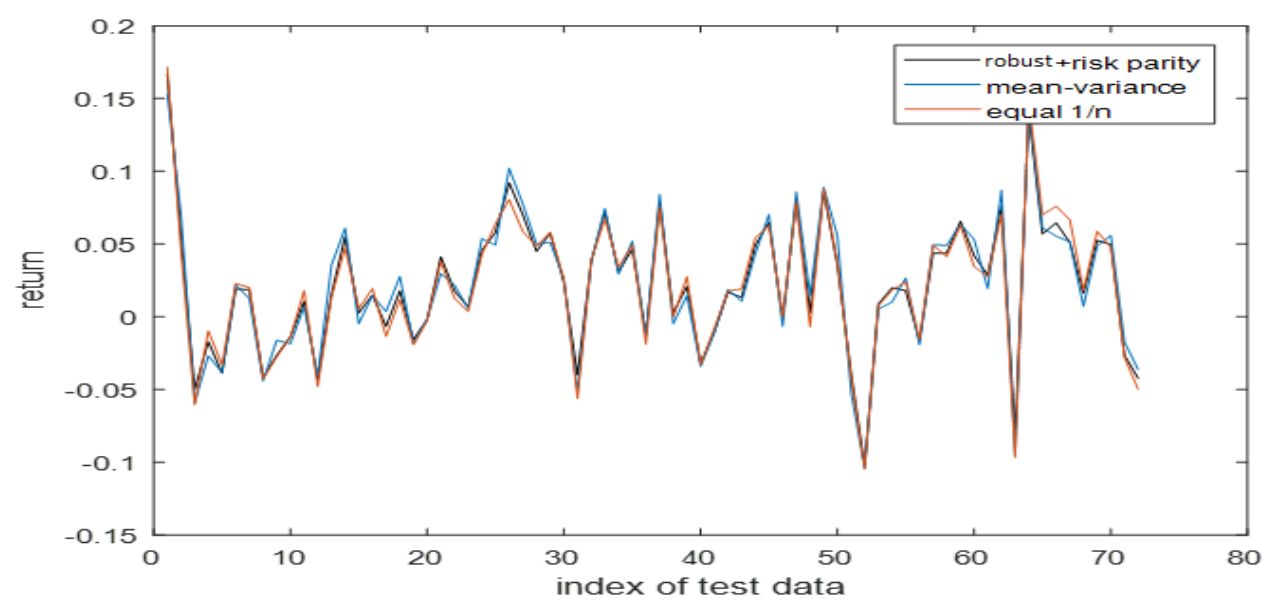

Figure 4. Performance of three portfolios on test data

To better observe the performance of the portfolios in severe market shortfalls, the graph is magnified at two points with negative returns, the result of which is shown in Figures (5) and (6).

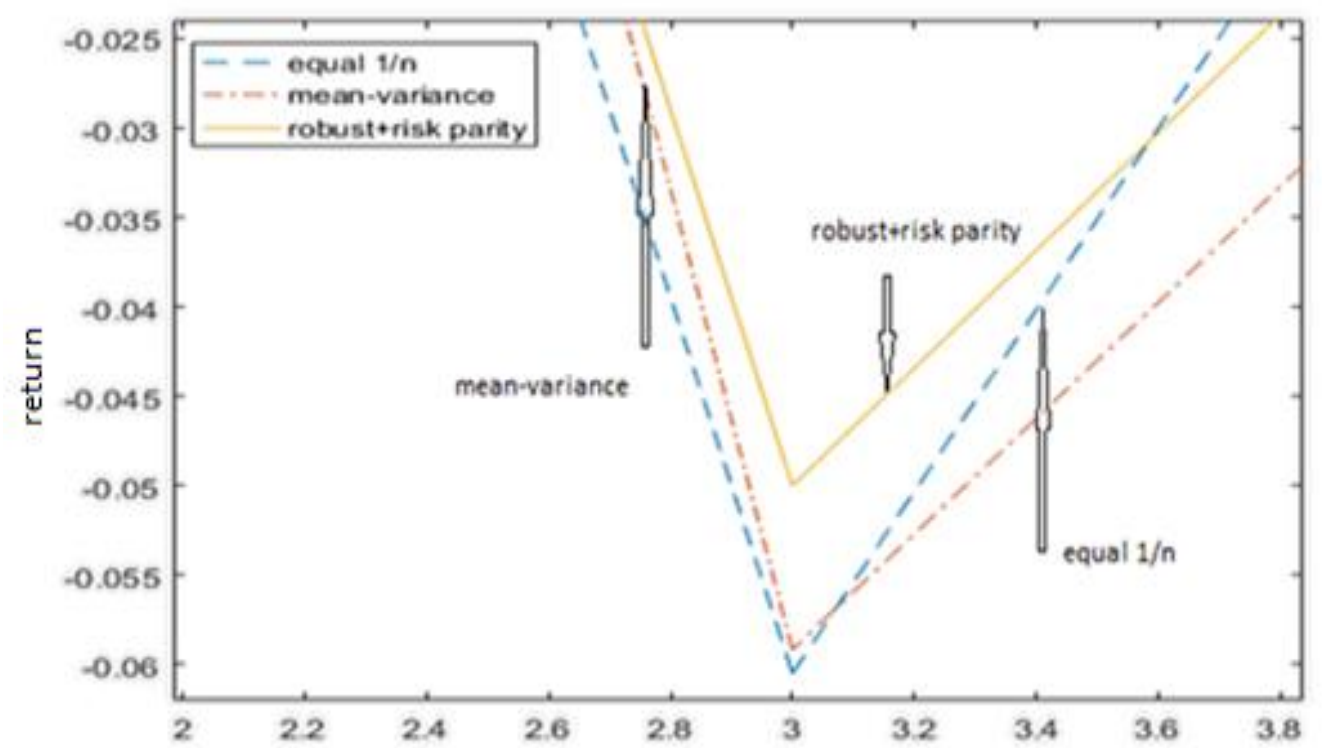


Figure 5. An example of the performance of portfolios in market shortfalls

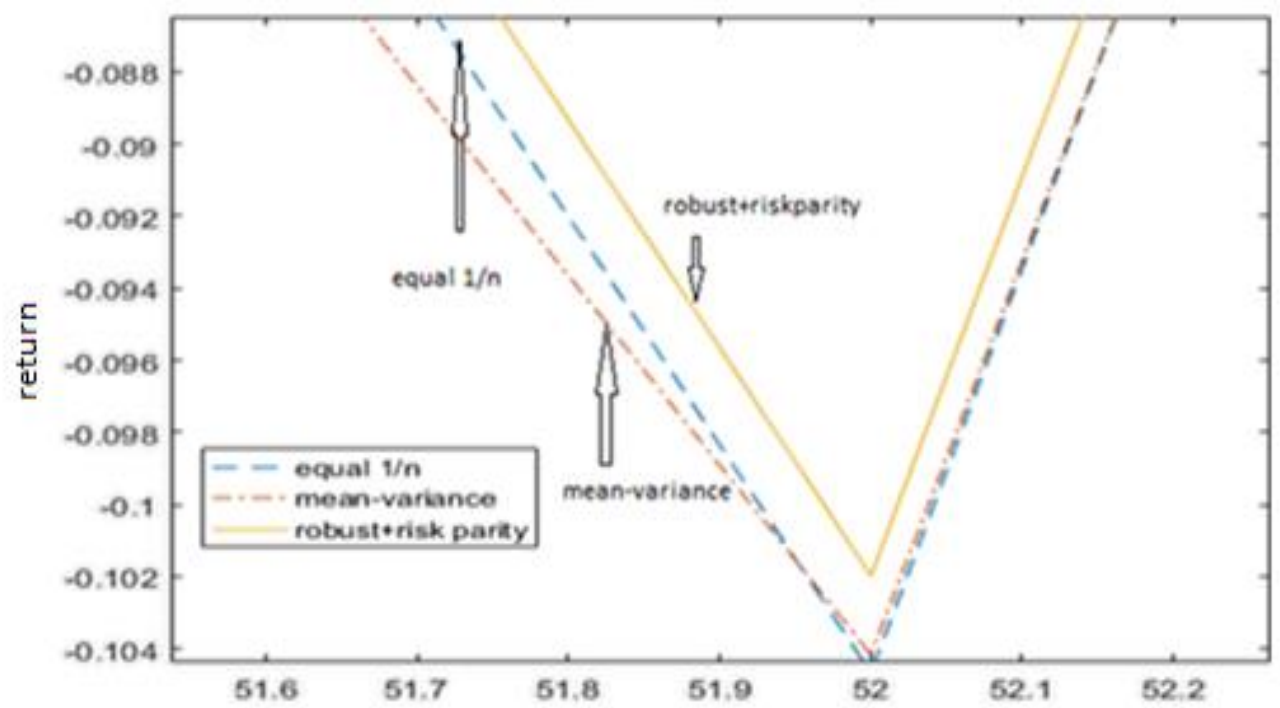

Figure 6. An example of the performance of portfolios in market shortfalls

As can be seen from these two examples, the research portfolio performs better. In general, for all market declines on test data, the performance of the three models is presented in Table (10).

Table 10. Performance of portfolios in market declines

\begin{tabular}{|c|c|}
\hline portfolio & Average weekly returns on markets declines \\
\hline Equal weighting & -0.0317 \\
\hline Markowitz & -0.0324 \\
\hline Relative robust risk parity & -0.028 \\
\hline
\end{tabular}

According to Table (10), the combined research model has experienced fewer declines than the other two models.

\section{Conclusion}

In the present study, the stock portfolio selection model with a combined approach of relative robust risk parity was introduced. In a risk parity portfolio, the weight of assets is such that the share of asset risk of the total portfolio risk 
is as equal as possible. This creates a kind of hedge for the portfolio against severe market declines. In the risk parity model, there is a covariance matrix parameter that can affect the stability of the problem. To establish the robustness of the optimal solution of the risk parity model, several scenarios based on historical data for the covariance matrix were considered and the objective function of the optimal risk parity portfolio was transformed into the worst relative scenario. Two main motivating questions of the present study are as follows: the first one examines the Sharpe ratio in the combined model of the research and compares it to the conventional mean-variance and equally weighted portfolio models and the second one investigates the resilience of this portfolio to market declines in relation compared to the afore-mentioned portfolios.

To fully identify the combined research method with an example and answer to these two questions, a stock portfolio consisting of eight assets (eight indices or industry) was formed from the Tehran Stock Exchange in the period 2011 to 2020. Using the index means forming a diverse portfolio of stocks in that industry. The time horizon of the stock portfolio is one week (closed for a period of one week) and 5 working days are considered for every week. 432 weekly returns were divided into 360 data for training and approximation of model parameters and 72 data for testing and evaluating stock portfolio performance. Then, portfolio performance on test data was calculated in terms of average return, risk and Sharp ratio criteria.

The results show that this hybrid portfolio with a Sharpe ratio of 0.504 has better performance than the other two models. It can be construed from the table that it is more resistant to market declines and can improve this value to 0.0037 on average and on a weekly scale. This conclusion is broadly consistent with the findings of Costa and Kwon (2018), Chaves et al. (2010), Maillard et al. (2009).

Because, like these researches, the present study shows that the Sharp ratio of the risk parity portfolio model is higher than the Markowitz and equal weight models.

Sharpe ratio represents the additional amount of return that an investor receives per unit of increase in risk. Due to the higher Sharpe ratio of the combined research model compared to the weight parity and mean-variance models, the use of the introduced combined model, especially in risky situations, is recommended to investors and portfolio managers to make the portfolio more resistant to severe losses. It should be noted that evaluating the combined model on test data for the portfolio and increasing scenarios for the covariance matrix over time can increase investors' confidence in the expected results. Future researchers are also encouraged to compare the performance of 
the combined model of the present study with other stock portfolio selection models, such as models based on the value at risk and expected shortfall. Also, another issue that can be considered for future research in this field is the presentation of a multi-period form of relative robust risk parity portfolio selection model.

\section{References}

Bai, Xi., Scheinberg, K., Tutuncu, R. (2015). Least-squares approach to risk parity in portfolio selection. Quantitative Finance, 16, 1-20.

Boyd, S., Kim, S. (2015). Robust efficient frontier analysis with a separable uncertainty model, [Stanford University], available: http://www.stanford. edu / boyd/papers/pdf/rob_ef_sep.pdf.

Caporin, M., Lisi, F., \& Janin, M. (2012 (. A survey on four families of performance measures, Working papers series, 1-26.

Chaves, D. B., Hsu, J. C., LI, F., \& Shakernia, O. (2011). Risk parity portfolio vs. other asset allocation heuristic portfolios. Journal of Investing, 20, 108-118.

Chopra, V.K., Ziemba, W.T. (1993). The effect of errors in means, variances, and covariances on optimal portfolio choice. J. Portfolio Manag, 19 (2), 6-11.

Costa, G., Kwon, R. (2018). Risk parity portfolio optimization under a Markov regime-switching framework, Quantitative Finance, 1-19.

Davallou, M., Fadaei. H., \& Herkhani. A. (2018). Stock allocation strategy with equal risk contribution. Guidelines of financial management, 1-30. (In Persian).

Demigueil, V., GarlappiI, L., \& Uppal, R. (1915-1953, 2009). Optimal versus naive diversification: How Inefficient is the 1/N Portfolio Strategy?. Review of Financial Studies, 22, 1916-1953.

Fabio, B., Francesco, C., \& Christian, C. (2019). Risk parity with expectiles. European Journal of Operational Research. 291(3), 1149-1163.

Gambeta, V., Kwon, R . (2020). Risk Return Trade-Off in Relaxed Risk Parity Portfolio Optimization, Journal of Risk and Financial Management, MDPI, Open Access Journal, 13(10), 1-28.

Gharakhani, M., Sadjadi, S., \& Safari, E. (2016). Robust portfolio optimization using CAPM. Journal of Production and Operations Management, 4, 93-102.

Gregory, C., Darby-Dowman, K. \& Mitra, G. (2016). Robust optimization and portfolio selection. European Journal of Operation Research, 212 (2), 417-428.

Groetzner, P., Werner, R. (2021). Multi-objective Optimization Under Uncertainty: A Multi-objective Robust (Relative) Regret Approach. European Journal of Operational Research.1-15.https://doi.org/10.1016/j.ejor.2021.03.068.

Khajehzadeh, M., Jafaria, M. (2015). Robust Fuzzy Multi-Objective Optimization Model for Portfolio Selection. Decision Engineering Journal, 1 (1), 31-56. 
Lee, W. (2011). Risk-Based Asset Allocation: A New Answer to an Old Question?. The Journal of Portfolio Management, 37(4), 11-28.

Maillard, S., Roncalli, T., \& Teiletche, J. (2009). On the properties equally weighted risk contributions portfolio. The Journal of Portfolio Management, 1-23.

Marat. M. (2020). A Modified Hierarchical Risk Parity Framework for Portfolio Management. Journal of Financial Data Science, Forthcoming, Available at SSRN: https://ssrn.com/abstract $=3588908$.

Maxey, D. (2015). Are Risk-Parity Funds a Better Strategy for Diversification?. The Wall Street Journal. https://www.wsj.com/articles/are-risk-parity-funds-abetter-strategy-for-diversification-1446805981.

Mohammadi, S., Mohammadi, E. (2018). Robust portfolio optimization based on minimax regret approach in Tehran stock exchange market. Journal of Industrial and Systems Engineering, 11(Special issue: 14th International Industrial Engineering Conference), 51-62.

Namdarzangeneh. S., Hassanpour, A. (2017). Robust optimization for multi-objective portfolio selection problem with min-max goal programming approach. Sharif Journal of Industrial Engineering \& Management, 1(33), 11-19.

Pflug, G., Wozabal, D. (2016). Ambiguity in portfolio selection. Quantitative Finance \& Accounting, 4, 435-442.

Rezaei, M., Ghahtarani, AR., \& Najafi,A. (2017). Application of Robust Optimization in Portfolio Selection Problem Through the Use of Conditional Drawdown at Risk. Journal of Operational Research and Its Applications, 14 (2), 81-93.

Roncalli, T. (2013). Introduction to risk parity and budgeting. 1st edition. Chapman \& Hall/CRC, USA.

Roncalli, T., Weisang, G. (2016). Risk parity portfolios with risk factors. Quant. Finance, 16(3), 377-388.

Simões, G., McDonald, M., Williams, S., Fenn, D., \& Hauser. R. (2018). Relative Robust Portfolio Optimization with benchmark regret. Quantitative Finance, 26, 1991-2003.

Bibliographic information of this paper for citing:

Mirmohammadi, Sayed Mohammad Ebrahi; Madanchi zaj, Mehdi; Panahian, Hossein and Jabbary, Hossein (2021). Stock Portfolio Optimization Using a Combined

Approach of Relative Robust Risk Parity. Iranian Journal of Finance, 5(4), 87-106.

Copyright (c) 2021, Sayed Mohammad Ebrahim Mirmohammadi, Mehdi

Madanchi zaj, Hossein Panahian, Hossein Jabbary 
Stock Portfolio Optimization Using a Combined Approach of...

| 107

(c) 